\title{
MBR 35 Editorial
}

\section{Justin Oakley ${ }^{1}$}

Published online: 7 July 2018

(c) Monash University 2018

In this issue, comprising Monash Bioethics Review volume 35, we present contributions on four topics of current debate in the field, along with a review of a new book on the problems undermining US government efforts to promote evidence-based medical practice and policy. In our first contribution to this issue of the journal, Dominic Wilkinson, Stavros Petrou, and Julian Savulescu provide a valuable new analysis of three different cost-effectiveness thresholds that can be utilised in determining the appropriateness of intensive care treatment. The second article, by Jonathan Anomaly, addresses the controversial topic of eugenics, and argues for several public policy proposals that aim to facilitate children being born with traits which give them a good chance of thriving in their lives. Public policy on reproduction is also the focus of our third article in this issue. In this article, Ryan Tonkens argues that a requirement for those seeking assisted reproduction to undergo a criminal background check and a child protection order, as is currently the law in Victoria, is unjustly discriminatory against infertile people. The fourth contribution to this issue, by Victoria Saigle and Eric Racine, provides a useful scoping review of the variety of ethical difficulties confronted by health professionals caring for patients who are suicidal, and identifies three key challenges facing practitioners in this area.

Justin Oakley

justin.oakley@monash.edu

1 Monash University, Melbourne, Australia 